\title{
Princípios gerais de criocirurgia no tratamento de tumores em pequenos animais: revisão de literatura
}

\author{
General principles of cryosurgery in the treatment of tumors in \\ small animals: literature review
}

\section{Principios generales de criocirugía en tratamiento de tumores en pequeños animales: revisión de la literatura}

\section{Genilson Fernandes de Queiroz'; Julia Maria Matera²}

Departamento de Clínicas Veterinárias da Universidade Estadual de Londrina, Londrina, PR, Brasil

\section{Resumo}

Objetivo: Divulgar a técnica, equipamentos utilizados, mecanismo de ação, indicações, vantagens e desvantagens, complicações e/ou seqüelas da criocirurgia no tratamento de lesões. Fontes Pesquisadas: O levantamento das informações foi realizado nos sistemas de bases de dados do CAB e MEDLINE num período retrospectivo de 30 anos. Síntese dos Dados: A criocirurgia consiste no tratamento de lesões por meio do seu congelamento (nitrogênio líquido a $-196^{\circ} \mathrm{C}$ ). Ela tem sido utilizada na medicina para o tratamento de neoplasias de pele, fígado, rins, próstata e cavidade oral. Na medicina veterinária ainda é pouco difundida e a literatura carece de informações quanto à sua aplicação. É um procedimento de fácil execução, no entanto, requer conhecimento da técnica, suas indicações, possíveis complicações e/ou seqüelas. Conclusões: A criocirurgia possibilita o tratamento de tumores de pele e/ou partes moles, principalmente a exérese de neoplasias malignas sem possibilidade de realização com margem de segurança por técnica cirúrgica convencional.

Palavras-chave: Criocirurgia. Crioterapia. Congelamento. Hipotermia induzida. Criogênicos. Nitrogênio. Neoplasias. Animais domésticos. 


\section{Mecanismo de Ação da Criocirurgia}

\section{Efeitos diretos}

A ação do gelo nos tecidos está relacionada aos efeitos diretos nas células e na estase vascular que se desenvolve após o descongelamento ${ }^{8}$. Durante o processo de congelamento ocorre a formação de cristais de gelo intracelular e extracelular ${ }^{6,17}$. A formação do gelo extracelular altera o gradiente osmótico entre o meio intracelular e o extracelular produzindo um efeito hiperosmolar que extrai água do meio intracelular e induz uma concentração tóxica de eletrólitos, a qual provoca um dano irreversível à célula. $\mathrm{O}$ aumento da concentração intracelular de eletrólitos resulta em alterações do $\mathrm{pH}$ nas macromoléculas e na membrana da célula $^{7,10,13,14,17,25-28}$.

A formação do gelo intracelular é dependente do tempo de congelamento e de uma temperatura mínima atingida. Um congelamento rápido à temperatura baixa resulta numa maior quantidade de gelo intracelular ${ }^{7,28}$. Desse modo, não ocorre perda de água para o meio extracelular e, na tentativa de manter o equilíbrio, formam-se pequenos cristais intracelulares ${ }^{3,10,13,14,27,29}$, os quais provocam danos nas organelas como as mitocôndrias e retículo endoplasmático induzindo a uma destruição celular irreversível. Quanto maior o tamanho dos cristais de gelo, maior o dano induzido por eles ${ }^{7,28}$.

\section{Efeitos indiretos sobre a circulação}

A injúria criogênica provoca estase vascular e anóxia tecidual resultando em necrose isquêmica ${ }^{7}$. A estase vascular está restrita ao local de exposição do criógeno e ocorre devido ao aumento da permeabilidade dos vasos resultando em hemoconcentração ${ }^{27}$. A formação de trombos ocorre em $65 \%$ dos capilares e 35 a $40 \%$ das arteríolas e vênulas a uma temperatura que varia de 3 a $11^{\circ} \mathrm{C}$, no entanto, a trombose completa dos vasos é detectável à temperatura de $-15 \mathrm{a}-20^{\circ} \mathrm{C}$ após 30 minutos de congelamento ${ }^{7}$.
Por meio de exame microscópico constatou-se que aproximadamente duas horas após o congelamento já se observa edema, danos focais aos capilares, hemorragia e formação de microtrombos. Por volta de 5 a 8 horas observa-se necrose focal ou segmentar dos vasos sangüíneos. Clinicamente a gangrena aparece

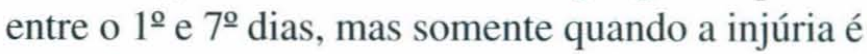
severa ${ }^{7}$.

\section{Efeitos imunológicos}

Acredita-se que as substâncias antigênicas normalmente encontradas nas células e liberadas quando ocorre destruição celular pelo congelamento estejam envolvidas na resposta antigênica ${ }^{17}$.

Uma resposta imunológica após tratamento com criocirurgia foi primeiro sugerida por volta dos anos 60 e 70, quando se observou a presença de anticorpos circulantes contra tecido prostático e adrenal de humanos e coelhos após congelamento ${ }^{7,11}$.

Em estudos experimentais demonstrou-se que linfócitos de animais com neoplasias tratadas com a crioterapia, quando transfundidos em animais com neoplasias semelhantes apresentaram um efeito citotóxico maior do que aqueles transfundidos a partir de animais nã- tratados. Esta resposta parece ser específica para cada tipo de tecido, sendo estimulada pela liberação de antígenos específicos do tumor durante ou após o congelamento ${ }^{30}$.

\section{Temperatura e Tempo de Congelamento- Descongelamento}

Os tecidos congelam a uma temperatura de $-0,6^{\circ} \mathrm{C}$, no entanto, esta temperatura não é letal. Os vários tipos celulares da pele apresentam comportamento distinto em relação à tolerância ao frio. Os melanócitos são os mais sensíveis ao frio e morrem em temperaturas que variam de $-4 \mathrm{a}-7^{\circ} \mathrm{C}$. As células das glândulas sebáceas e folículos pilosos são sensíveis a temperaturas abaixo de $-20^{\circ} \mathrm{C}$, os queratinócitos apresentam uma resistência considerável, morrem entre -20 
e $-30^{\circ} \mathrm{C}$ e o tecido conjuntivo é o mais resistente, pois fibroblastos, morrem entre $-30 \mathrm{e}-35^{\circ} \mathrm{C}^{5,7}$.

Os cirurgiões determinaram que a temperatura mínima para produzir crionecrose está entre $-20 \mathrm{a}-30^{\circ} \mathrm{C}$, ocorrendo uma acentuada destruição quando a temperatura está em torno de -40 a $-60^{\circ} \mathrm{C}^{10}$.

Muitos cirurgiões utilizaram a temperatura de $-20^{\circ} \mathrm{C}$ como temperatura-alvo para o tratamento de tumores malignos, no entanto, estudos mais recentes têm demonstrado que esta temperatura não é adequada, exceto para tumores benignos. No tratamento crioterápico de tumores do tipo carcinoma basocelular e carcinoma de células escamosas, as técnicas atuais requerem temperaturas no tecido-alvo em torno de $-50^{\circ} \mathrm{C}$, temperatura na qual ocorre congelamento total da pele ${ }^{8}$. Os cirurgiões do Reino Unido recomendam um congelamento contínuo de 30 segundos, já nos Estados Unidos preconiza-se um congelamento intermitente por 30 a 60 segundos até que sensores térmicos registrem temperatura de $-50^{\circ} \mathrm{C}$ ou obtenha-se um halo de congelamento de $5 \mathrm{~mm}$ ao redor do tumor ${ }^{5}$.

O tempo de descongelamento do halo deve ser de pelo menos 1 minuto, no entanto, o descongelamento completo ocorre por volta de 3 a 5 minutos ${ }^{5}$.

\section{Ciclos de Congelamento- Descongelamento}

A destruição celular é diretamente proporcional ao número de ciclos de congelamento-descongelamento. Desse modo, durante um procedimento de criocirurgia, via de regra deve-se congelar o tecido-alvo pelo menos duas vezes ${ }^{10}$. Repetidos ciclos de congelamento-descongelamento asseguram o máximo dos efeitos destrutivos em tumores cutâneos ${ }^{7,8,28}$.

As células cancerígenas são resistentes ao congelamento. A capacidade de resistência destas células é a razão para a utilização de repetidos ciclos de congelamento-descongelamento ${ }^{31}$.

\section{Indicações da Criocirurgia}

A utilização da criocirurgia na medicina teve aplicação clínica substancial por volta da década de 90 , no tratamento de tumores cutâneos. O surgimento de aparelhos de ultra-som intra-operatório possibilitou a sua utilização em tumores de próstata, fígado e rins, permitindo a visualização do processo de congelamento ${ }^{31}$.

Diversos tipos de tumores em diversas áreas do corpo como pele, osso, ânus, reto, útero e cavidades têm sido tratados com criocirurgia ${ }^{31}$, podendo ser utilizada no tratamento de lesões benignas, tumores malignos e pré-malignos. É o método considerado como tratamento de escolha ou tratamento alternativo em diversas doenças de pele. Qualquer área do corpo pode ser tratada, não existe limite de idade ${ }^{8,32}$. Possibilita o tratamento de tumores recidivos, lesões próximas ou fixadas a ossos e cartilagens, tumores grandes ou pequenos. É indicada para pacientes cirúrgicos de alto risco, portadores de marca-passo, de coagulopatia e idosos. É um método alternativo para pacientes nos quais outros métodos de tratamento são impraticáveis ${ }^{8}$. Na medicina veterinária é mencionada para o tratamento de tumores da cavidade oral e nasal ${ }^{6,10,11,13,14}$ cutâneos e/ou de tecidos moles ${ }^{6,9-11,13,14,33,34}$ (Figura 2), tumores $^{6,10,11,13,14,35}$ e fístulas perianais e mamárias ${ }^{6,10,11} \mathrm{sem}$ relatos objetivos de sua eficácia. 


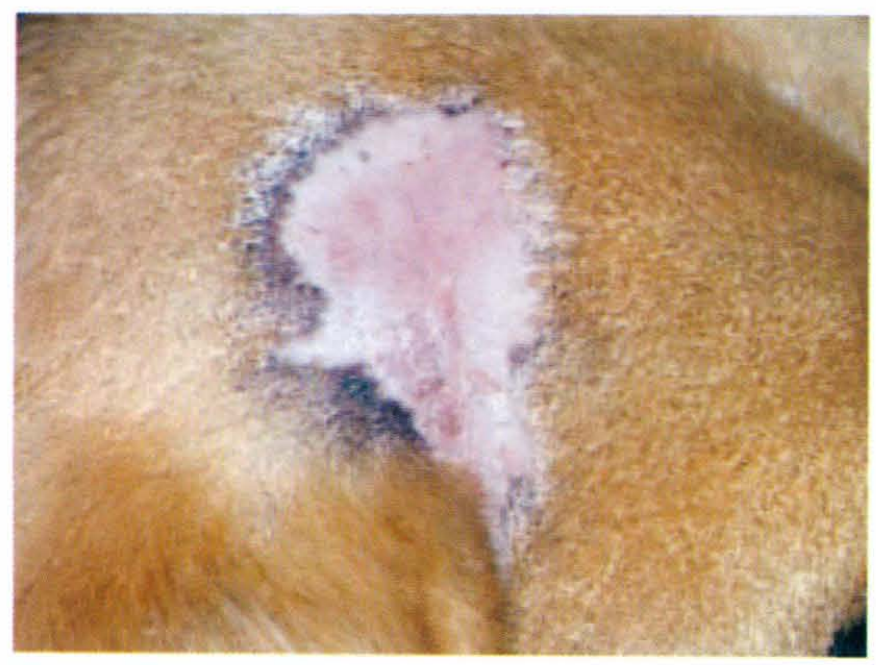

Figura 3 - Alopecia e hipopigmentação em região cefálica de cão após 45 dias do procedimento de criocirurgia. Serviço de Cirurgia de Pequenos Animais - HOVET-FMVZ/USP

\section{Complicações, Contra- Indicações e Efeitos Adversos}

A incidência de complicações após a criocirurgia é baixa. Elas podem resultar de erro de tratamento, falha no equipamento, ou inexperiência do operador. As complicações podem ser classificadas como permanentes ou temporárias. As complicações temporárias mais freqüentes incluem hemorragias, edema ou dor durante ou imediatamente após o tratamento. As complicações permanentes incluem retração tecidual, neuropatia, ruptura de tendões, alopecia, ectrópio e hipopigmentação $0^{8,28,36}$.

As contra-indicações podem ser caracterizadas como absolutas e relativas. Há poucas contraindicações absolutas, elas incluem urticária induzida pelo frio, crioglobulinemia, criofibrinogenemia (presença de criofibrinogênio no sangue, tipo anormal de fibrinogênio encontrado raramente no plasma que é precipitado por resfriamento, mas dissolve-se novamente quando aquecido à temperatura ambiente) $)^{28,32,36}$.

Os efeitos adversos consistem em cicatrização lenta, cicatrizes extensas, disfunção estética e funcional importante, persistência de linfoadenopatia. Apesar desses possíveis efeitos, isso não deve interferir na escolha da técnica quando a indicação for adequada ${ }^{37}$.

\section{Pós-Operatório}

A reação tecidual que ocorre após a criocirurgia é previsível e a cicatrização ocorre por segunda intenção. Uma exsudação local inicia-se nas primeiras 24 horas e continua por diversos dias, nessa fase recomenda-se uma lavagem da ferida com água e sabão $0^{5,26}$. Em áreas que reagem com forte edema e eritema após a criocirurgia indica-se a utilização imediata de cremes esteroidais para minimizar estas reações ${ }^{8,32}$. Alguns autores recomendam a injeção intramuscular de fosfato de betametasona 30 minutos antes do tratamento, seguido pela prednisona oral durante 3 dias $^{8}$.

A cicatrização procede lentamente com formação do tecido de granulação e formação de crosta. Nessa fase os cuidados consistem em lavagem da ferida, aplicação de pomada com antibiótico, e se necessário, faz-se debridamento ${ }^{26}$. O tempo de cicatrização varia conforme o tamanho e localização da lesão, podendo alterar-se de 4 a 14 semanas $^{26}$.

\section{Vantagens e Desvantagens da Criocirurgia}

\section{Vantagens}

A criocirurgia é uma técnica de tratamento relativamente rápida ${ }^{6,13,14,38}$, de fácil aplicação ${ }^{38}$, de baixo custo $^{8,38}$ e segura, evitando, dessa forma, os riscos da anestesia, principalmente para animais idosos $s^{6,8,10}$. Apresenta um menor potencial de invasão e menor morbidade quando comparada com a intervenção cirúrgica ${ }^{39}$, podendo ser utilizada em lesões em áreas da pele onde não há tecido suficiente para aproximar; ou em lesões grandes, onde não é possível o fechamento com sutura ${ }^{6,11,40}$.

\section{Desvantagens}

O custo inicial por equipamento varia de 700 a 1.000 dólares, no entanto, este investimento pode ser compensado na utilização de anestésicos, material cirúrgico e o tempo de procedimento ${ }^{38}$. Dependendo do número de procedimentos realizados semanalmente, $o$ 
desperdício de nitrogênio líquido pela evaporação pode ser um inconveniente ${ }^{38}$. Não é um procedimento cirúrgico limpo, mas se o proprietário é avisado previamente sobre a ação do congelamento nos tecidos, poucas reclamações surgirão ${ }^{1,6,38}$. utilizada em órgãos como fígado, rins, próstata, lembrando que nestes locais requer utilização de equipamentos apropriados. Embora ela seja de fácil execução requer conhecimento da técnica, suas indicações, possíveis complicações e/ou seqüelas.

\section{Considerações Finais}

A criocirurgia é uma técnica de fácil execução que possibilita o tratamento de tumores de pele e/ou de partes moles, principalmente os localizados em regiões onde não há possibilidade do uso da margem de segurança na sua excisão e sem tecido suficiente para aproximação dos bordos da ferida cirúrgica. Ela pode ainda ser

\section{Agradecimento}

À Fundação de Amparo à Pesquisa do Estado de São Paulo (FAPESP) pelo apoio, na forma de bolsa, para o desenvolvimento da presente pesquisa.

\section{Abstract}

Objective: To disclose the technique, equipment, mechanism of action, indications, advantages and disadvantages, complication and/or after-effects of cryosurgery in treatment of lesions. Data Sources: Data search was carried out in database systems of CAB and MEDLINE over a 30-year term. Data Synthesis: Cryosurgery consists of the treatment of lesions through freezing (liquid nitrogen at $-196^{\circ}$ C). It has been used in medicine for the treatment of skin, liver, kidneys, prostate and oral cavity neoplasias. In veterinary medicine it is still not broadly known and literature lacks information as to its application. It is an easily performed procedure, requiring however knowledge of the technique, of indications and possible complications and after-effects. Conclusions: Cryosurgery enables treating skin and/or soft tissues tumors, mainly extraction of malign neoplasms with no possibility of using safety margin by conventional surgical technique.

Keywords: Cryogenic surgery. Cryotherapy. Freezing techniques. Hyperthermia, induced. Cryogenics. Nitrogen. Neoplasms. Pets. 


\section{Resumen}

Objetivo: Divulgar la técnica, los equipos utilizados, el mecanismo de acción, las indicaciones, las ventajas y desventajas, las complicaciones y/o secuelas de la criocirugía en el tratamiento de lesiones. Fuentes Pesquisadas: Se obtuvieron las informaciones en los sistemas de bases de datos del CAB y MEDLINE en un periodo retrospectivo de 30 años. Síntesis de los Datos: La criocirugía consiste en el tratamiento de lesiones a través de su congelamiento (nitrógeno líquido $a-196^{\circ} \mathrm{C}$ ). Viene utilizándose en la medicina para el tratamiento de neoplasias de piel, hígado, riñones, próstata y cavidad oral. En la medicina veterinaria aun está muy poco difundida y la literatura carece de informaciones respecto de su aplicación. Es un procedimiento de fácil ejecución, entretanto, requiere conocimiento de la técnica, de sus indicaciones, de las posibles complicaciones y/o secuelas. Conclusiones: La criocirugía posibilita el tratamiento de tumores de piel y/o tejidos blandos, principalmente la extirpación de neoplasias malignas sin posibilidad de realización con un margen de seguridad por la técnica convencional.

Palabras-clave: Criocirugía. Crioterapia. Congelamiento. Hipotermia inducida. Criogénicos. Nitrógeno. Neoplasmas. Animales domésticos.

\section{Referências}

1. PODKONJAK, K. R. Veterinary cryotherapy. 1. A comprehensive look at uses, principles and success. Veterinary Medicine and Small Animal Clinician, v. 77, p. 51-64, 1982.

2. WHITTAKER, D. K. Mechanisms of tissue destruction following cryosurgery. Annals of the Royal College of Surgeons of England, v. 66, p. 313-318, 1984.

3. ZACARIAN, S. A. Cryosurgery in the management of cutaneous disorders and malignant tumors of the skin. Comprehensive Therapy, v. 20, p. 379401, 1994.

4. COOPER, S. M.; DAWBER, R. P. R. The history of cryosurgery. Journal of the Royal Society of Medicine, v. 94, p. 196-201, 2001.

5. GRAHAM, G. F. Cryosurgery in the management of cutaneous malignancies. Clinics in
Dermatology, v. 19, p. 321-327, 2001.

6. GREINER, T. P. et al. Cryosurgery. Veterinary Clinics of North America: Small Animal Practice, v. 5 , p. 565-581, 1975.

7. ZOUBOULIS, C. C. Principles of cutaneous cryosurgery: an update. Dermatology, v. 198, p. 111-117, 1999.

8. KUFLIK, E. G. Cryosurgery updated. Journal of the American Academy of Dermatology, v. 31, p. $925-944,1994$.

9. KRAHWINKEL, D. J. Cryosurgical treatment of skin diseases. Veterinary Clinics of North America: Small Animal Practice, v. 10, p. 787-801, 1980.

10. BOJRAB, M. J. Veterinary cryosurgery: an overview. Norden News, v. 53, p. 16-20, 1978. 
11. GOLDSTEIN, R. S.; HESS, P. W. Cryosurgery of canine and feline tumors. Journal of the American Animal Hospital Association, v. 12, p. 340-349, 1976.

12. GOLDSTEIN, R. S.; HESS, P. W. Cryosurgical treatment of cancer. Veterinary Clinics of North America: Small Animal Practice, v. 7, p. 51-64, 1977.

13. LANE, J. G. Practical cryosurgery: an introduction for small animal clinicians. Journal of Small Animal Practice, v. 15, p. 715-725, 1974.

14. LANE, J. G. The clinical applications of cryosurgery in small animal practice. The Veterinary Annual, v. 14 , p. 216-219, 1974.

15. MAMEDE, R. C. M. Princípios gerais e técnicas de criocirurgia em cabeça e pescoço. In: BRANDÃO, L. G.; FERRAZ, A. R. Cirurgia de cabeça e pescoço. São Paulo: Roca, 1989. p. 689.

16. FERRIS, D. G.; HO, J. J. Cryosurgical equipment: a critical review. Journal of Pharmacy Practice, v. 35, p.185-193, 1992.

17. LEOPARD, P. J. Cryosurgery, and it's application to oral surgery. British Journal of Oral Surgery, v. 13 , p. $128-152,1975$.

18. GOURLEY, I. M.; VASSEUR, P. B. General small animal surgery. Philadelphia: Lippincott, 1985. p. $929-939$

19. HOYT JR., R. F.; SEIM III, H. B. Veterinary cryosurgery: mechanism of cell death, cryosurgical instrumentation, and cryogens. Part I. The Compendium on Continuing Education for the Practicing Veterinarian, v. 3, p. 426-432, 1981.

20. MAIA, M.; RIBEIRO, A. E. Curso de criocirurgia. São Paulo: Centro de Estudos Dermatológicos Adolpho Carlos Lindenberg, Clínica de Dermatologia da Santa Casa de São Paulo, 1997. 19 p. Apostila.

21. SEIM III, H. B.; HOYT JR., R. F. Veterinary cryosurgery: principles of application. Part II. The Compendium on Continuing Education for the Practicing Veterinarian, v. 4, p. 695-702, 1982.

22. GAGE, A. A. Cryosurgery of advanced tumors. Clinics in Dermatology, v. 8, p. 86-95, 1990.

23. GAGE, A. A. Cryosurgery in the treatment of cancer. Surgery, Gynecology and Obstetrics, v. 174, p. 73-92, 1992.

24. WESHAHY, A. H. Intralesional cryosurgery: a new technique using cryoneedles. Journal of Dermatology and Surgery Oncology, v. 19, p. 123-126, 1993.

25. HOLDEN, H. B.; McKELVIE, P. Cryosurgery in the treatment of head and neck neoplasia. British Journal Surgery, v. 59, p. 709-712, 1972.

26. KUFLIK, E. G. Cryosurgery for cutaneous malinancy. Dermatology Surgery, v. 23, p. 10811087, 1997.

27. SEIM III, H. B. Mechanisms of cold-induced cellular death. Veterinary Clinics of North America: Small Animal Practice, v. 10, p. 755-762, 1980.

28. THAI, K. E.; SINCLAIR, R. D. Cryosurgery of benign skin lesions. Australasian Journal of Dermatology, v. 40, p. 175-186, 1999.

29. WITHROW, S. J. Cryosurgery of canine and feline tumors. Journal of the American Animal Hospital Association, v. 11, p. 271-282, 1975.

30. MARQUES, L. A. C. Criocirurgia: nossa experiência. Rio de Janeiro, 1989. Dissertação (Mestrado) - Pontifícia Universidade Católica do Rio de Janeiro. 
31. GAGE, A. A.; BAUST, J. G. Cryosurgery: a review of recent advances and current issues. Cryoletters, v. 23, p. 69-78, 2002.

32. ZOUBOULIS, C. C. Cryosurgery in dermatology. European Journal of Dermatology, v. 8, p. 466-474, 1998.

33. CLARKE, R. E. Cryosurgical treatment of feline cutaneous squamous cell carcinoma. Australian Veterinary Practitioner, v. 21, p.148-153, 1991.

34. LANA, S. E. et al. Feline cutaneous squamous cell carcinoma of the nasal planun and the pinnae: 61 cases. Journal of the American Animal Hospital Association, v. 33, p. 329-332, 1997.

35. LISKA, W. D. Anorectal and perianal cryosurgery. Veterinary Clinics of North America: Small Animal Practice, v. 10, p. 803-820, 1980.
36. YOUNG, R.; SINCLAIR, R. Practical cryosurgery. Australian Family Physician, v. 26, p. 1045-1047, 1997.

37. MARCIANI, R. D.; TRODAHL, M. J. Postoperative sequelae of cryosurgery. Journal of Oral Surgery, v. 33, p. 458-461, 1975.

38. WITHROW, S. J. Why worry about cancer in pets? In: WITHROW, S. J.; MacEVEN, E. G. Small animal clinical oncology. 3. ed. Philadelphia: Saunders, 2001. p. 1-3.

39. HOFFMANN, N. E.; BISCHOF, J. C. Cryosurgery of normal and tumor tissue in the dorsal skin fala chambre: Part 1. Termal response. Journal of Biomechanical Engineering, v. 123, p. 301-309, 2001.

40. SCOTT, D. W. et al. Small animal dermatology. Philadelphia: Saunders, 1995. 1213 p. 